\title{
Brain activation during mental rotation in school children and adults
}

\author{
K. Kucian ${ }^{1}$, M. von Aster ${ }^{2,3}$, T. Loenneker ${ }^{1,4}$, T. Dietrich ${ }^{1}$, F. W. Mast ${ }^{5}$, E. Martin ${ }^{1}$ \\ ${ }^{1}$ MR-Center, University Children's Hospital, Zurich, Switzerland \\ ${ }^{2}$ Department of Child and Adolescent Psychiatry, University of Zurich, Zurich, Switzerland \\ ${ }^{3}$ Department of Child and Adolescent Psychiatry, St. Joseph Hospital, Berlin, Germany \\ ${ }^{4}$ Center for Integrative Human Physiology, University of Zurich, Zurich, Switzerland \\ ${ }^{5}$ Institute of Psychology, University of Lausanne, Lausanne, Switzerland
}

Received: April 24, 2006 / Accepted: October 30, 2006 / Published online: December 11, 2006

(C) Springer-Verlag 2006

\begin{abstract}
Summary Mental rotation is a complex cognitive skill depending on the manipulation of mental representations. We aimed to investigate the maturing neuronal network for mental rotation by measuring brain activation in 20 children and 20 adults using functional magnetic resonance imaging. Our results indicate that brain activation patterns are very similar between children and adults. However, adults exhibit stronger activation in the left intraparietal sulcus compared to children. This finding suggests a shift of activation from a predominantly right parietal activation in children to a bilateral activation pattern in adults. Furthermore, adults show a deactivation of the posterior cingulate gyrus and precuneus, which is not observed in children. In conclusion, developmental changes of brain activation during mental rotation are leading to a bilateral parietal activation pattern and faster performance.
\end{abstract}

Keywords: Brain function, children, cognitive development, fMRI, gender differences, intraparietal sulcus (IPS)

Abbreviations: $R T$ Reaction time, $A C C$ accuracy rate, $P C G$ posterior cingulate gyrus, IPS intraparietal sulcus, $\Delta S$ signal change

\section{Introduction}

The investigation of cognitive development in infants and children provides a fascinating reflection of human brain development. A wealth of brain mapping work has been performed in adults, yet cognitive development in children has not been studied to such a degree. The topography and extent of cerebral activation associated with a particular task may alter as children become more proficient. Concomitant changes can be observed in intensities of cerebral

Correspondence: Karin Kucian, MR-Center, University Children's Hospital, Steinwiesstrasse 75, 8032 Zurich, Switzerland

e-mail: karin.kucian@kispi.unizh.ch activation associated with task performance (Rivkin, 2000; Stiles et al., 2003).

In this study, the development of brain areas for visuospatial processing was examined using a mental rotation task. Mental rotation is a complex cognitive ability, which is supported by a variety of neuropsychological functions such as shape perception, spatial reasoning and problem solving. In adults, brain activation during mental rotation is associated with the parietal lobes, as well as with regions of the frontal and occipital lobe (Alivisatos and Petrides, 1997; Carpenter et al., 1999; Harris et al., 2000; Jordan et al., 2002; Kosslyn et al., 1998; Richter et al., 2000, 1997; Tagaris et al., 1996; Thomsen et al., 2000; Vingerhoets et al., 2001; Weiss et al., 2003). The hypothesis that the parietal lobes, most often centered on the intraparietal sulci, are implicated in mental rotation is supported by different neuroimaging methods. However, it is not conclusive which role the right and the left IPS plays for mental rotation. Most studies examining mental rotation using functional magnetic resonance imaging (fMRI), positron emission tomography (PET) or event related potentials (ERP) report bilateral parietal activation (Carpenter et al., 1999; Cohen et al., 1996; Desrocher et al., 1995; Jordan et al., 2002; Kosslyn et al., 1998; Peronnet and Farah, 1989; Richter et al., 1997; Tagaris et al., 1996; Wijers et al., 1989). However, a transcranial magnetic stimulation (TMS) study found that only disrupting neural activity in the right parietal lobe interfered with mental rotation performance (Harris and Miniussi, 2003). Moreover, a PET study examining mental rotation revealed right IPS activation only 
(Harris et al., 2000). In contrast, a 85 year old man exhibited severe dysfunction in mental rotation after a stroke involving the left angular gyrus and left parietal lobe (Carota et al., 2004). Furthermore, two independent PETstudies engendered specific activity for mental rotation only within the left parietal lobe (Alivisatos and Petrides, 1997; Vingerhoets et al., 2001). Finally, Jordan et al. (2002) reported activation of the left IPS in men and bilateral IPS activation in women. Based on behavioral responses, i.e. reaction time and accuracy, several surveys report males to outperform females in mental rotation tasks (Crucian and Berenbaum, 1998; Halpern, 2000; Linn and Petersen, 1985). However, only few neuroimaging studies have evaluated gender differences in mental rotation and the results are highly inconsistent (Dietrich et al., 2001; Jordan et al., 2002; Roberts and Bell, 2000; Tagaris et al., 1996; Thomsen et al., 2000; Unterrainer et al., 2000; Weiss et al., 2003). In a previous study, we found significant differences in brain activation but no differences in performance between women and men during the same mental rotation task we used in the present study (Kucian et al., 2005).

Only recently, neuroimaging studies have begun to examine the neural underpinnings of mental rotation in children. To our knowledge, four neuroimaging studies have been carried out until now. One study used functional magnetic imaging (fMRI) to examine brain activation of 912 year old children during mental rotation of alphanumeric stimuli (Booth et al., 1999, 2000). Results showed that healthy children and adults activate similar brain areas, but there were differences in the distribution of activation across these networks. Adults showed more activation in the superior parietal and middle frontal areas and less activation in the supramarginal gyrus (Booth et al., 2000). In children, the inferior parietal lobe was more active in the right hemisphere, whereas activation in the superior parietal lobe was more lateralized in the left hemisphere (Booth et al., 1999). Booth et al. (1999) reported no lateralization bias in adults. Taken together, these fMRI studies revealed overlapping patterns of activation in children and adults, but certain regions associated with visuospatial skills may be more lateralized in children. Two other studies in children used EEG and focused on the relationships between gender, age and hemispheric activation during different mental rotation tasks (Roberts and Bell, 2000, 2002). One of these studies (Roberts and Bell, 2000) showed that men exhibited more activation (lower EEG power values) compared to women in left parietal and posterior temporal regions, whereas boys' and girls' power values did not differ in these regions in a two-dimensional (2D) mental rotation task (gingerbread man figures). Moreover, they observed faster reaction times for men compared to women, whilst there were no differences between boys and girls. However, the results of the other EEG study (Roberts and Bell, 2002) indicated gender differences not only in adults but also in children. Men and boys exhibited greater left parietal activation than women and girls in the $2 \mathrm{D}$ mental rotation task (gingerbread man figures). When using a different 2D stimulus (alphanumeric characters), men unlike women, exhibited left posterior temporal activation whereas there were no differences between boys and girls. During a 3D mental rotation task (basketball players), all participants exhibited greater activation in right than in left parietal areas, but no gender differences in adults or children could be found (Roberts and Bell, 2002). Taken together, neuroimaging studies in children provide some evidence that the parietal lobe plays an important role in development and gender differences revealed in cerebral patterns during mental rotation.

Compared to EEG, fMRI techniques offer the advantage to investigate cortical activations with higher spatial resolution and thus provide the ability to focus on developmental changes in location and strength of brain activation. The purpose of the present fMRI study is to examine brain areas during mental rotation in children and adults. We aim to provide further insights into the maturation of brain areas involved in mental rotation and possible differences between genders. We expect children to activate similar brain areas as adults, yet differences in the distribution and levels of activation may reflect maturation and experience. Moreover, we assume that boys and girls will perform at similar levels comparable to our findings in adults.

\section{Materials and methods}

\section{Participants}

Twenty-six normally achieving school children and twenty-two adults participated in this study. Participants were healthy, right-handed volunteers with no psychiatric or medical complications as determined by a detailed questionnaire. None of the participants suffered from any neuroanatomical abnormality as determined by high resolution structural magnetic resonance scans. All participants were medication-free. Valid MR data without motion artifacts (less than half a voxel size) were obtained in 20 out of 22 adult participants (10 females and 10 males, mean age $27.2 \pm 5.0$ years). Six children had to be excluded from data analysis due to motion of more than half a voxel size ( 1 child), image artifacts ( 3 children) or poor behavioral performance ( 2 children). The remaining 20 children were split in two age groups: ten 3rd grade schoolchildren ( 5 females and 5 males, mean age $9.2 \pm 0.2$ years) and ten 6th grade schoolchildren ( 5 females and 5 males, mean age $12.0 \pm 0.3$ years). Written informed consent for the participation in the study was obtained from the adult participants and from the legal guardians of the children. The study was approved by the local ethics committee based on the World Medical Association's Declaration of Helsinki (WHO, Ethical Principles for Medical Research Involving Human Subjects, 2002). 

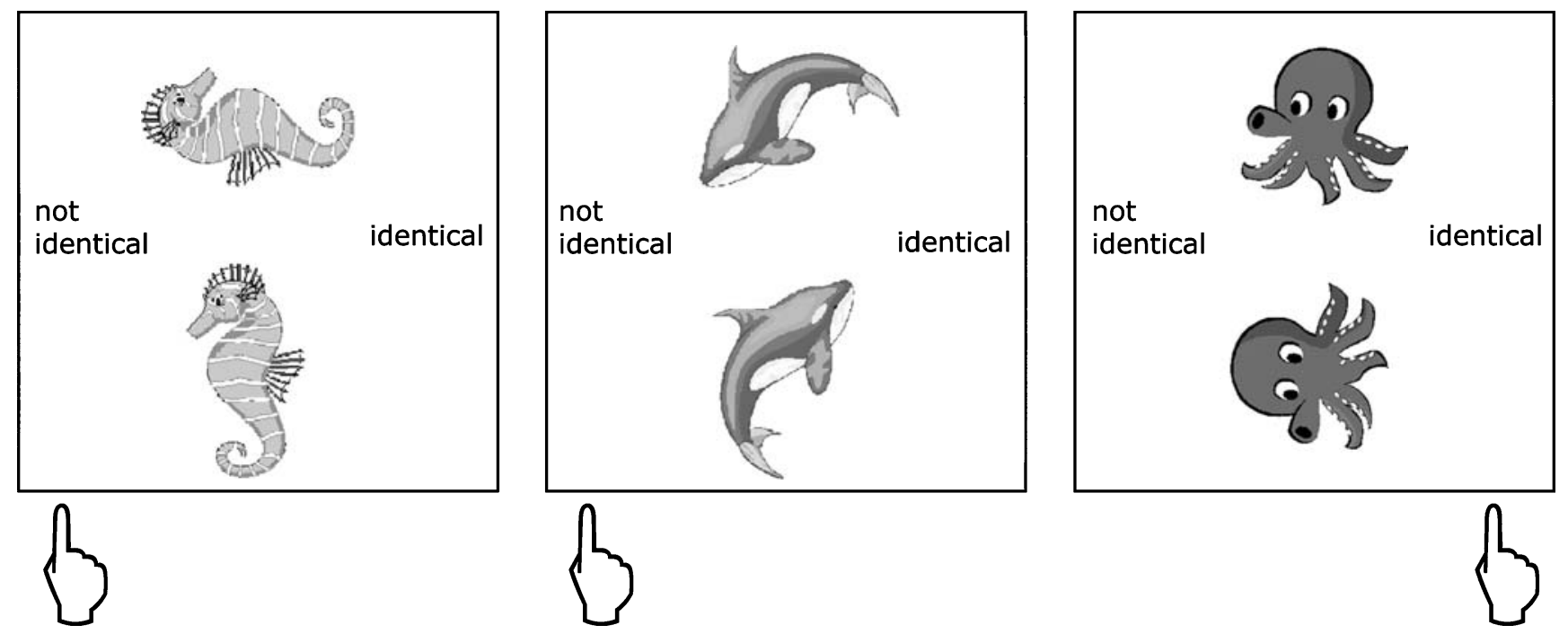

Fig. 1. Mental rotation paradigm. First block: seahorses; second block: whales; third block: octopi. Participants had to mentally rotate these 2D pictures either by $\pm 90^{\circ}$ or $180^{\circ}$ to decide whether the two animals were the same or different

\section{Behavioral tests in children}

All children underwent behavioral tests to assess their linguistic and mathematical competences prior to the study. Sixth grade children completed two modules of a test battery for semantic and linguistic verbal fluency in German and mathematical competence (Klassencockpit, Ausgabe 2003/2004). Two 6th grade children were excluded from the study due to poor test results with more than one standard deviation below the average performance of the 6th grade Swiss normative sample in one or both modules. Children of the $3 \mathrm{rd}$ grade were tested for number processing and calculation abilities [ZAREKI (von Aster, 2001)] and for reading and spelling skills [Knuspel's Leseaufgaben (Marx, 1998); Salzburger Rechtschreibtest (Landerl et al., 1997)]. The performance of all 3rd grade children was within the Swiss normative sample.

\section{Stimuli and task}

Before entering the scanner the participants were carefully instructed about the examination procedure and the task. The paradigm was a $2 \mathrm{D}$ mental rotation task (see Fig. 1). Two colored drawings of animals were presented simultaneously one above the other on a screen. The two stimuli appeared at varying orientations $\left(0^{\circ}, 90^{\circ}, 180^{\circ}, 270^{\circ}\right)$ whereby the difference in orientation between the two stimuli was either $90^{\circ}$ or $180^{\circ}$, but in varied presentation positions of the stimuli. Participants had to mentally rotate one of these pictures by either $\pm 90^{\circ}$ or $180^{\circ}$ in the picture plane to decide whether the two animals were the same or different. In a pilot study, four different stimuli types (seahorses, whales, octopi, fishes) were tested with eight 3 rd grade children ( 3 girls, 5 boys) and 11 adults ( 5 women, 6 men). The result of the pilot study ensured that the task difficulty was age appropriate; children reached a mean accuracy level of $80 \%$ with a mean reaction time of 3'492 ms. The three stimuli that were chosen for this study (seahorse, whale, octopus) revealed similar error rates and reaction times. Drawings of fishes were excluded as stimuli due to slightly higher error rates and slightly longer reaction times compared to the other three stimuli. The fishes were used later for the practice session prior to scanning.

Children who participated in the fMRI study were instructed to the paradigm in two separate steps outside the scanner to ensure that they fully understood the task. In a first step, the task was explained to them and the children practiced with cut out drawings of fishes so that they could manually rotate the objects for verification if they were identical or not. In a second step, the paradigm was performed on a desktop computer and children had to solve two to four $20 \mathrm{~s}$ blocks of practice trials by pressing the corresponding mouse button. Subjects were asked to work as quickly and as accurately as possible. After completing practice trials with a maximum of two errors within a block, we continued with the actual fMRI experiment.

During fMRI examination the computer-generated paradigm (E-Prime, Psychology Software Tools Inc.), synchronized by trigger pulses of the fMRI-sequence, was presented to the participants via video-goggles (Resonance Technology Inc., Northridge, U.S.A.). The paradigm was a classical box-car design consisting of three blocks of mental rotation tasks (first block: seahorses, second block: whales, third block: octopi). The blocks lasted $80 \mathrm{~s}$, separated by breaks of $12.5 \mathrm{~s}$. The maximum duration of the randomized self-paced stimuli presentation was $6 \mathrm{~s}$, separated by inter-stimulus intervals (ISI) of $1 \mathrm{~s}$. Participants were asked to fixate a point at the center of the screen during the rest phases. An additional $0^{\circ}$ rotation control condition was discarded to make the fMRI data acquisition less demanding for the young children. Furthermore, prior pilot testing revealed identical and accurate activation patterns compared to literature when contrasting mental rotation against fixation. Behavioral data were collected by means of a response box (LUMINA, Cedrus Corporation, San Pedro, U.S.A).

\section{fMRI image acquisition}

Functional MRI acquisition was performed on a 1.5 Tesla whole-body scanner (GE Medical Systems, Milwaukee, WI, USA), using gradientrecalled echo planar imaging (repetition time, $\mathrm{TR}=3.2 \mathrm{~s}$; echo time, $\mathrm{TE}=$ $55 \mathrm{~ms}$; flip angle $=90^{\circ}$; field of view $(\mathrm{FOV})=240 \mathrm{~mm} \times 240 \mathrm{~mm}$; matrix size $=64 \times 64$; voxel size $=3.75 \mathrm{~mm} \times 3.75 \mathrm{~mm} ; 32$ contiguous slices parallel to the AC-PC line, slice thickness $=4 \mathrm{~mm}$ ). Three-dimensional anatomical images of the entire brain were obtained by using a T1-weighted gradient echo pulse sequence $(\mathrm{TR}=27 \mathrm{~ms}$; FOV $=240 \mathrm{~mm} \times 240 \mathrm{~mm} \times 144 \mathrm{~mm}$; matrix size $=256 \times 192 \times 90)$.

\section{Data analysis}

The behavioral data were statistically analyzed using SPSS (SPSS 11.5, Chicago, U.S.A.). Statistical data analysis of behavioral results was based on 20 female participants (10 adults, 10 children) and 19 male participants 
( 9 adults, 10 children). One male participant had to be excluded because of a recording failure in behavioral data acquisition. The fMRI data were analyzed using the Statistical Parametric Mapping (SPM99) software (Wellcome Department of Cognitive Neurology, London, U.K.). The first four images were discarded thus allowing for a steady-state magnetization. All images were realigned and transformed into the standardized stereotactic reference system (EPI-template provided by the Montreal Neurological Institute). The small differences in time courses and locations of activation foci between child and adult brains validate the feasibility of direct statistical comparison of these groups within a common space (Kang et al., 2003). Each normalized scan was smoothed with a $9 \mathrm{~mm}$ full-width at half-maximum Gaussian kernel. Changes in regional blood oxygen level-dependent (BOLD) contrast were determined by applying the general linear model (GLM) to each voxel. A within-group voxel-wise comparison of regional BOLD change was performed using $t$-statistics to test hypotheses regarding significant changes in BOLD. The resulting set of voxel values for each contrast constituted a statistical parametric map of the $t$-statistic $(\operatorname{SPM}\{\mathrm{T}\})$. A second level analysis was performed on the basis of the linear contrasts for each participant and condition. Reported activated brain regions of random effects analysis and two-sample $t$-test analysis had been subjected to a family wise error (FWE) correction with a minimum number of 10 voxels. However, for not to run the risk of losing activation foci in children or differences in brain activation between the genders, also uncorrected thresholds were consulted. Finally, MNI coordinates (Montreal Neurological Institute) of activated voxels were transformed into the Talairach and Tournoux reference system.

Region of interest (ROI) definition, extraction of data for the region and statistical analysis of ROI data using SPM was performed by MarsBar (MarsBar Version 0.37, Matthew Brett). Activated clusters subjected to FWE correction with a minimum number of 10 voxels served as templates for ROI definition.

Lateralization index was computed by counting the total number of activated voxels on each hemisphere at $p<0.05$ FWE corrected and dividing the differences of the number of activated voxels on the right and on the left hemisphere by the total number of activated voxels [(\# voxels right - \# voxels left $) /(\#$ voxels right $+\#$ voxels left)]. Obtained results range from -1 to +1 . Negative values demonstrate left lateralized, positive values right lateralized and values near to zero bilateral activation patterns.

\section{Results}

\section{Behavioral results}

The accuracy levels for all groups were high (83\% in 3rd graders, $86 \%$ in 6 th graders, $87 \%$ in adults). Mean reaction times were $2811 \mathrm{~ms}$ for $3 \mathrm{rd}$ graders, $2728 \mathrm{~ms}$ for 6th graders and $2153 \mathrm{~ms}$ for adults. Table 1 shows the mean reaction time and accuracy rate and standard deviations for the mental rotation tasks separately for the two children groups and adults.

Multivariate GLM analysis was computed with reaction time (RT) and accuracy rate (ACC) as dependent variables and gender (female/male) and age (3rd grade/6th grade/adults) as fixed factors. The analysis indicated significant age differences $(\mathrm{F}(4,64)=2.663, p<0.05)$. Posthoc $t$-tests showed that both 3 rd grade $(p<0.01)$ and 6th grade children $(p<0.05)$ needed significantly more time to solve the mental rotation tasks than adults. However, we found no significant differences in ACC between 3rd graders, 6th graders and adults $(p>0.1)$. Moreover, there
Table 1. Behavioral results

\begin{tabular}{lrrr}
\hline & All & Females & Males \\
\hline 3rd grade children & & & \\
Reaction time (ms) & & & \\
$\quad$ Mean & 2810.8 & 2651.4 & 2970.1 \\
S.D. & 460.3 & 546.0 & 339.4 \\
Accuracy rate (\%) & & & \\
$\quad$ Mean & 83.1 & 83.0 & 83.2 \\
S.D. & 10.1 & 8.7 & 12.3 \\
6th grade children & & & \\
Reaction time (ms) & 2727.8 & 2873.0 & 2582.7 \\
Mean & 551.4 & 614.0 & 504.4 \\
S.D. & & & \\
Accuracy rate (\%) & 85.5 & 81.2 & 89.8 \\
$\quad$ Mean & 11.2 & 14.3 & 5.4 \\
S.D. & & & \\
Adults & & & \\
Reaction time (ms) & 2152.8 & 2331.3 & 1954.3 \\
Mean & 647.2 & 748.3 & 478.0 \\
S.D. & & & \\
Accuracy rate (\%) & 87.8 & 85.6 & 4.7 \\
$\quad$ Mean & 7.4 & 8.8 & \\
S.D. & & & \\
\hline
\end{tabular}

Means and standard deviations (S.D.) of reaction times and accuracy rates, split into sexes for 3rd grade and 6th grade children and adults. None of the comparisons between female and male participants turned out to be significant.

were no significant differences in ACC $(p \geq 0.08)$ between the three subsequent blocks of stimuli whether in children nor in adults. Children answered fastest during the first block $(p \leq 0.05)$ and adults needed significantly more time during the second block $(p \leq 0.05)$. Thus, we did not observe any effects of practice during the course of the experiment.

Neither accuracy nor reaction time revealed significant gender differences in adults or children $(\mathrm{F}(2,32)=1.069$, $p>0.1$ ). We computed the effect sizes (Cohen's d) and they ranged from small values, $d=0.21$ (ACC 3 rd graders), to high values, $d=0.90$ (ACC 6th graders) (Cohen, 1988).

The majority of the participants (79\%) needed more time to mentally rotate objects presented $180^{\circ}$ (mean RT children: $3038 \pm 788 \mathrm{~ms}$, mean RT adults: $2330 \pm 811 \mathrm{~ms})$ than $\pm 90^{\circ}$ (mean RT children: $2789 \pm 671 \mathrm{~ms}$, mean RT adults: $2245 \pm 806 \mathrm{~ms}$ ). However, the differences between participants were relatively large and the mean overall RTs ranged from 1195 to $4002 \mathrm{~ms}$. Paired $t$-tests revealed significantly higher RTs for $180^{\circ}$ than for $\pm 90^{\circ}$ rotation $(p<0.01)$ in children but did not reach significance in adults $(p=0.14)$. We calculated a quotient by dividing the mean RT for $180^{\circ}$ by the mean of RT for $\pm 90^{\circ}$ for each participant using the following equation: $\left[\mathrm{RT}_{180^{\circ}} /\right.$ $\left.\mathrm{RT}_{ \pm 90^{\circ}}\right]-1$. This formula results in a positive value for participants who needed more time for $180^{\circ}$ than for $\pm 90^{\circ}$ and a negative value for participants who needed more time 
for $\pm 90^{\circ}$ than for $180^{\circ}$ rotation. Using this normalization we then compared all participants in a one-sample $t$-test and the results showed that children $(p<0.001)$ as well as adults $(p<0.05)$ needed significantly more time to rotate objects for $180^{\circ}$ than for $\pm 90^{\circ}$. The fact that participants needed more time for $180^{\circ}$ than $90^{\circ}$ rotation, was indepen-
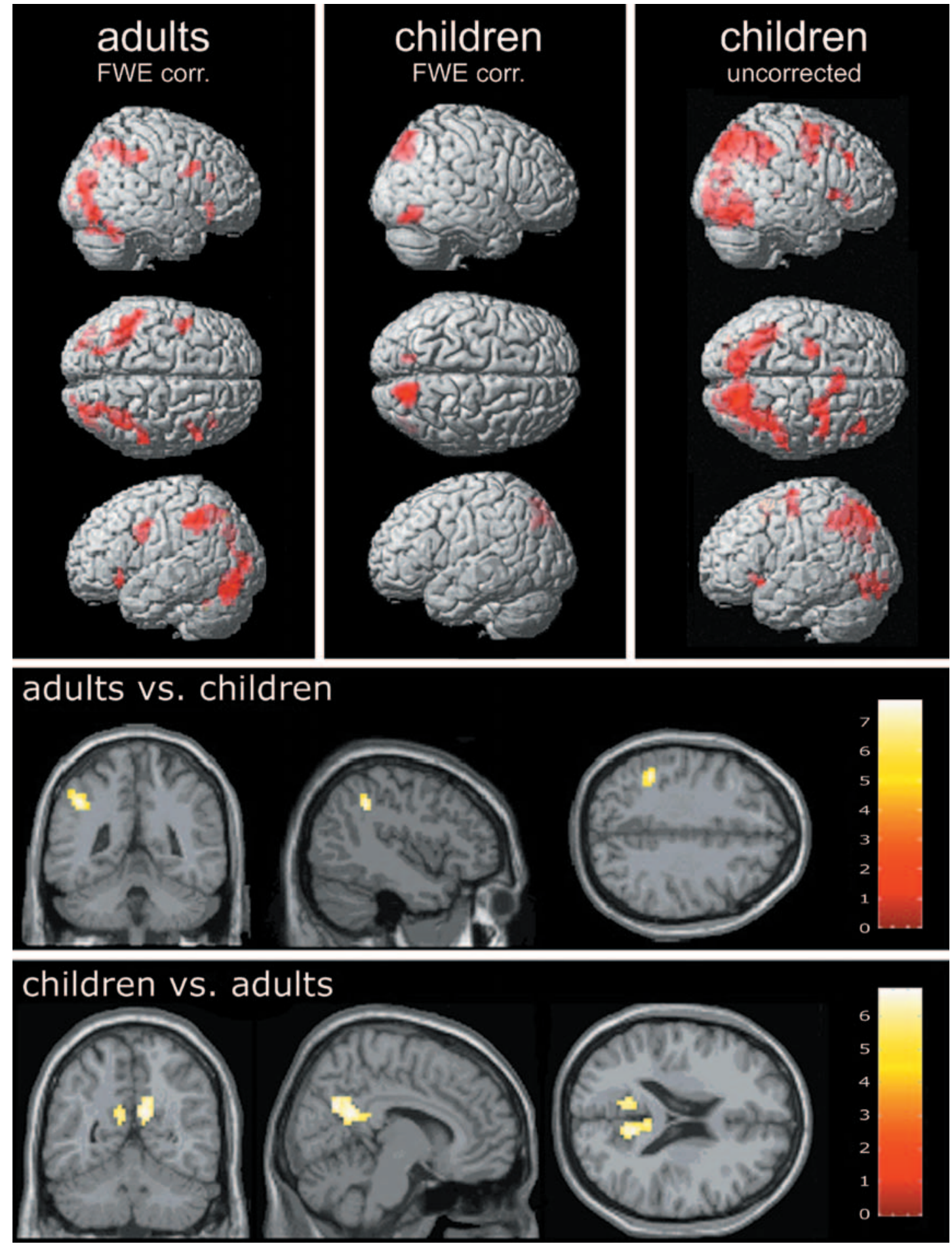

Fig. 2. Pattern of significant activation during mental rotation is depicted on a normalized brain for adults and children using FWE correction $(p=0.05$ FWE corrected, $T=11.73, k=10$ ). In addition, for better visualization of activated networks in children, activation pattern is also shown for uncorrected values $(p=0.0001$ uncorrected, $T=10.55, k=10)$. Areas of significant activation are illustrated on template brain sections of the contrast adults versus children ( $p=0.05$ FWE corrected, $T=6.84, k=10)$ at the global maximum (Talairach coordinates: $9,-51,22)$ and of the contrast children versus adults $(p=0.05$ FWE corrected, $T=7.69, k=10)$ at the global maximum (Talairach coordinates: $-42,-42,38$ ) using SPM 
dent from stimulus presentation. Paired $t$-test indicated that all combinations requiring $180^{\circ}$ rotation (' $0{ }^{\circ}$ and $180^{\circ}$ '; ' $90^{\circ}$ and $270^{\circ}$ ') evoked longer RTs compared to $90^{\circ}$ rotation (' $0{ }^{\circ}$ and $180^{\circ}$ ' vs. $90^{\circ}, p<0.01$; ' $90^{\circ}$ and $270^{\circ}$ ' vs. $\left.90^{\circ}, p<0.01\right)$.

\section{fMRI results}

Children

The brain activation patterns of the children resembled those of the adults when using uncorrected $p$-values in children.
Main areas of activation relative to fixation ( $p=0.0001$ uncorrected) in children included in both hemispheres the precuneus, intraparietal sulcus, superior parietal lobe, fusiform gyrus, thalamus, middle frontal gyrus (BA6 and BA9), superior frontal gyrus (BA6), inferior frontal gyrus (BA47), insula and only on the left hemisphere the inferior occipital gyrus (BA17) (Fig. 2 'children uncorrected', Table 2). When using the corrected threshold of significance $(p=0.05 \mathrm{FWE}$ corrected ) we found fewer areas of activation and a more right lateralized activation pattern in children compared to adults (Lateralization index $=0.821$ ). Only peak voxels of

Table 2. Cortical activation of adults and children

\begin{tabular}{|c|c|c|c|c|c|}
\hline \multirow[t]{2}{*}{ Anatomical region } & \multicolumn{3}{|c|}{ Talairach coordinates } & \multirow[t]{2}{*}{$T$} & \multirow[t]{2}{*}{ Volume $k_{\mathrm{E}}$} \\
\hline & $x$ & $y$ & $z$ & & \\
\hline \multicolumn{6}{|l|}{ Adults $(p=0.05$ FWE corrected $)$} \\
\hline Left inferior parietal lobe, intraparietal sulcus (BA40) & -45 & -41 & 42 & 11.73 & 534 \\
\hline Left fusiform gyrus (BA19) & -39 & -76 & -11 & 10.79 & \\
\hline Right inferior parietal lobe, intraparietal sulcus (BA40) & 36 & -47 & 41 & 11.57 & 434 \\
\hline Right middle occipital gyrus (BA19) & 30 & -84 & 18 & 10.27 & \\
\hline Right fusiform gyrus (BA19) & 21 & -80 & -19 & 9.75 & 106 \\
\hline Left inferior frontal gyrus (BA47) & -33 & 23 & -9 & 9.42 & 38 \\
\hline Right inferior frontal gyrus (BA9) & 39 & 7 & 22 & 9.40 & 48 \\
\hline Right inferior frontal sulcus (BA9) & 50 & 16 & 27 & 9.01 & \\
\hline Right inferior frontal sulcus (BA46) & 39 & 27 & 18 & 9.31 & 12 \\
\hline Right inferior frontal gyrus (BA47) & 33 & 29 & -6 & 8.83 & 35 \\
\hline Left inferior frontal gyrus (BA9) & -42 & 4 & 25 & 8.33 & 72 \\
\hline Right cuneus (BA18) & 21 & -93 & 7 & 7.85 & 12 \\
\hline Left fusiform gyrus (BA37) & -33 & -54 & -23 & 7.85 & 11 \\
\hline \multicolumn{6}{|l|}{ Children $(p=0.05$ FWE corrected $)$} \\
\hline Right superior parietal lobe (BA7) & 18 & -71 & 42 & 10.55 & 181 \\
\hline Right fusiform gyrus (BA19) & 36 & -76 & -9 & 8.41 & 71 \\
\hline Left superior parietal lobe (BA7) & -15 & -68 & 39 & 8.02 & 26 \\
\hline \multicolumn{6}{|l|}{ Children $(p=0.0001$ uncorrected $)$} \\
\hline Right precuneus (BA7) & 18 & -71 & 42 & 10.55 & 2519 \\
\hline Right superior parietal lobule (BA7) & 12 & -64 & 56 & 9.22 & \\
\hline Right fusiform gyrus (BA19) & 36 & -76 & -9 & 8.41 & \\
\hline Left precuneus (BA7) & -15 & -68 & 39 & 8.02 & \\
\hline Right intraparietal sulcus (BA40) & 39 & -42 & 33 & 6.80 & \\
\hline Left intraparietal sulcus (BA40) & -39 & -47 & 41 & 6.60 & \\
\hline Right pulvinar & 12 & -26 & 4 & 7.14 & 167 \\
\hline Right thalamus & 6 & -17 & 1 & 7.00 & \\
\hline Left pulvinar & -15 & -26 & 4 & 5.60 & \\
\hline Left thalamus & -9 & -17 & 6 & 5.28 & \\
\hline Right middle frontal gyrus (BA6) & 27 & 0 & 58 & 6.70 & 401 \\
\hline Right superior frontal gyrus (BA6) & 3 & 14 & 55 & 6.28 & \\
\hline Left fusiform gyrus (BA19) & -24 & -82 & -6 & 6.63 & 166 \\
\hline Left inferior occipital gyrus (BA17) & -12 & -91 & -8 & 5.34 & \\
\hline Left superior frontal gyrus (BA6) & -21 & -8 & 67 & 6.32 & 119 \\
\hline Left middle frontal gyrus (BA6) & -27 & -6 & 50 & 6.27 & \\
\hline Right insula & 24 & 26 & -4 & 5.84 & 60 \\
\hline Left inferior frontal gyrus (BA47) & -42 & 17 & -8 & 5.82 & 27 \\
\hline Left insula & -36 & 23 & -6 & 5.34 & \\
\hline Right inferior frontal gyrus (BA47) & 50 & 15 & 2 & 5.64 & 20 \\
\hline Right middle frontal gyrus (BA9) & 42 & 31 & 32 & 5.24 & 44 \\
\hline
\end{tabular}

Anatomical localization, Talairach coordinates, $T$ scores of significant activated clusters and cluster size of adults and children at different statistical levels. 
the activation pattern in children maintained a FWE correction (bilateral superior parietal lobe, but stronger pronounced on the right hemisphere, right fusiform gyrus, Fig. 2 'children FWE corr.', Table 2).

\section{Adults}

Adults showed significant ( $p=0.05$ FWE corrected) bilateral activation during mental rotation (relative to fixation) in the inferior parietal lobule (BA40) including the intraparietal sulcus (IPS) (Fig. 2 'adults FWE corr.', Table 2). Significant activation was also found bilaterally in visual areas (BA18, BA19), extrastriate cortex and fusiform gyrus. Frontal activation was found in the inferior frontal gyrus (BA47, BA9) bilaterally, extending on the right hemi-

Table 3. Cortical activation of adults vs. children

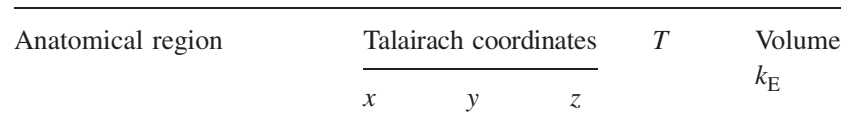

\section{Children-adults}

Right posterior cingulate gyrus (BA31)

Right precuneus (BA31)

Right precuneus (BA31)

Left precuneus (BA31)

Left posterior cingulate

gyrus (BA31)

Adults-children

$\begin{array}{llllll}\text { Left inferior parietal lobe, } & -42 & -42 & 38 & 7.69 & 49\end{array}$

intraparietal sulcus

Anatomical localization, Talairach coordinates, $T$ scores of significant activated clusters and cluster size of "children-adults" and "adultsChildren" at $p=0.05$ FWE corrected.

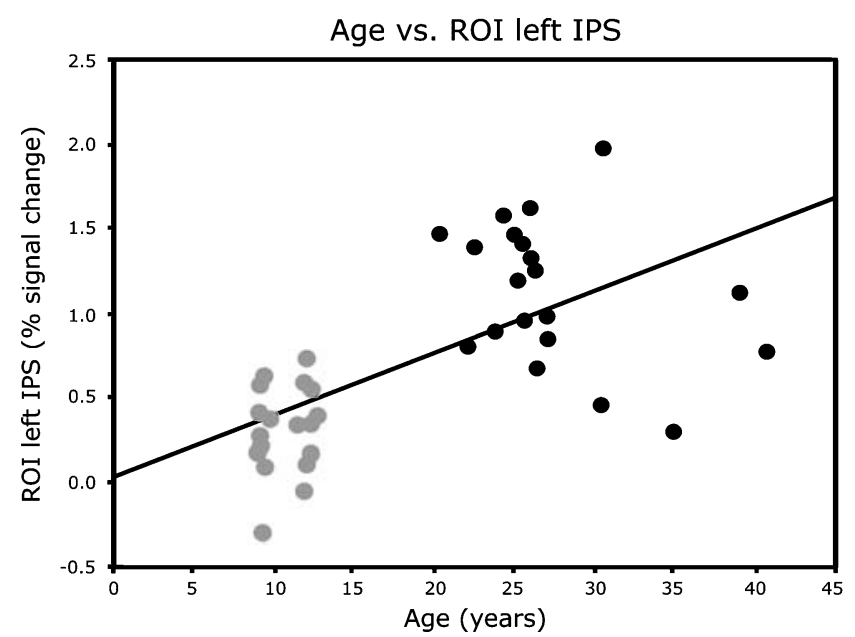

sphere into the inferior frontal sulcus. Observed activation pattern was bilateral (Lateralization index $=0.002$ ).

Children vs. adults

Although there was substantial overlap in the activated pattern during mental rotation in adults and children, the calculated contrasts revealed significant differences (Table 3). The contrast 'children vs. adults' revealed more activation in the posterior cingulate gyrus (PCG) and precuneus bilaterally ( $p=0.05$ FWE corrected) (Fig. 2 'children vs. adults'). Post-hoc ROI analysis of percentage of signal change $(\Delta S)$ in these clusters revealed a deactivation in adults (mean $\Delta S=-0.57$ ) in contrast to children (mean $\Delta S=0.02$ ).

Adults vs. children

Figure 2 'adults vs. children' shows activated clusters when comparing adults with children. Adults showed more activation in the left IPS ( $p=0.05$ FWE corrected). ROI analysis of $\Delta \mathrm{S}$ in the left IPS supported stronger activation in adults (mean $\Delta \mathrm{S}=1.13$ ) compared to children (mean $\Delta \mathrm{S}=0.31)$.

3rd grade vs. 6th grade children

Both contrasts, 3rd graders vs. 6th graders and 6th graders vs. 3rd graders revealed no significant differences in brain activation.

\section{Integration of behavioral and fMRI data}

Since reaction times were markedly different between children and adults, we calculated a correlation between per-

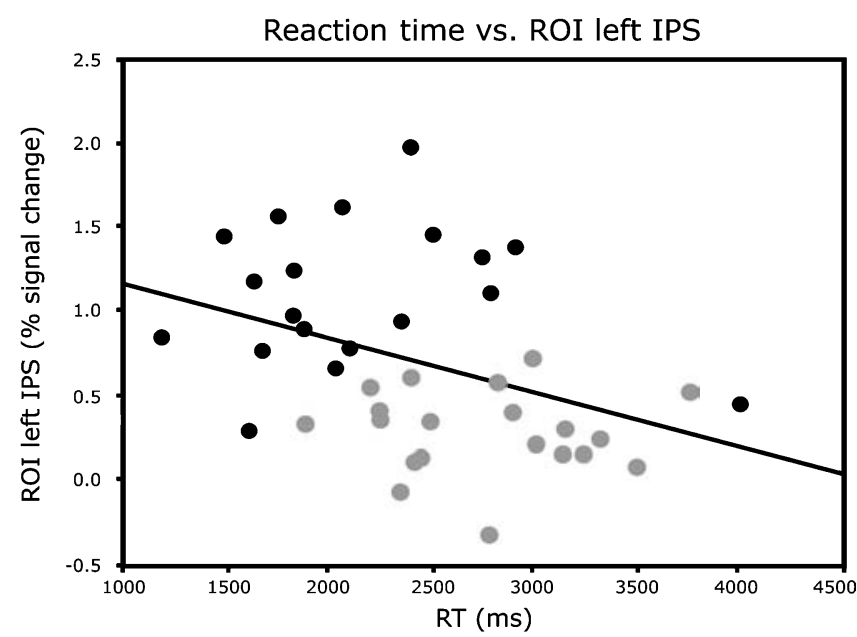

Fig. 3. Correlation of $\%$ signal change in the left IPS with age $(r=0.633, p<0.001)$ and RT $(r=-0.384, p<0.05)$. Gray dots $=$ children, black dots $=$ adults 
centage of signal change in the left IPS and RT. The left IPS is the main region that showed a weaker activation in children compared to adults. A ROI analysis in the left IPS showed a significant correlation between the percentage signal change in this region with RT and age (Fig. 3). Activation in the left IPS increased with age (Pearson correlation $r=0.633, p<0.001)$ and decreased with longer RTs (Pearson correlation $r=-0.384, p<0.05$ ). However, no significant correlations between left IPS activation and age (children: $r=0.104, p<0.7$; adults: $r=-0.339, p<0.2$ ) or RT (children: $r=-0.048, p<0.9$; adults: $r=-0.017$, $p<1.0$ ), respectively, could be found when splitting children and adults.

In addition, after partialing for accuracy, reaction time and number of stimuli, significant correlation was obtained between percentage of signal change in the left IPS and age $(r=0.658, p<0.0001)$.

\section{Gender differences}

For the analysis of the effect of gender in children, the brain activation in girls was subtracted from that in boys and vice versa. Both contrasts yielded no gender differences in activation ( $p=0.001$ uncorrected, $k=7)$. We also found no difference between girls and boys when splitting them into two groups of different ages (3rd grade children and 6th grade children) and analyzing them separately.

In contrast, differences in brain activation between adult women and men were found (Kucian et al., 2005). In particular, women showed stronger activation in the right middle temporal gyrus, the right inferior frontal gyrus and the left primary motor cortex compared to men $(p=0.001$ uncorrected, $k=5$ ).

\section{Discussion}

The purpose of this study was to examine the brain activation pattern in children during mental rotation and to compare it to that of adults using fMRI. Of special interest to us was, whether there are differences in performance between children and adults and if so, how they are related to brain activation patters.

Our results indicated changes in the lateralization of active brain regions known to play a relevant role in mental rotation of objects in space as a consequence of development.

\section{Developmental outcome}

We found a significant difference in left IPS activation between children and adults. The activation in the IPS was weaker in children than in adults. A critical issue in developmental cognitive neuroscience is the extent to which the functional neuroanatomy underlying task performance differs in adults and children (Schlaggar et al., 2002). The importance of taking performance differences into account has been noted in several developmental neuroimaging studies (Casey, 2002; Gaillard et al., 2001; Klingberg et al., 2002). We found similarly high rates of correct responses in children and adults and concluded that both groups were able to perform the task equally well. Moreover, possible influences of error trials on brain activation are very small due to the low error rates, which were absolutely comparable in children and adults (Murphy and Garavan, 2004). Even though there was a wide range of RTs, the individual performance level did not correlate with brain activation in the left IPS within the children and adult groups. In addition, developmental differences in the left IPS were still obtained after controlling for accuracy, reaction time and number of stimuli. Data presented in this study suggest that stronger brain activation in the left IPS is more likely to reflect maturation rather than individual mental rotation ability.

One could argue that the observed differences in brain activation between children and adults reflect different $\operatorname{cog}$ nitive strategies instead of developmental characteristics. However, this explanation seems rather unlikely given the behavioral results of this study. On the one hand, both children and adults showed comparable accuracy rates and, on the other hand, both needed more time to assess the correct answer when rotating the two-dimensional stimuli by $180^{\circ}$ compared to $90^{\circ}$. This difference in time suggests that participants not only used mental rotation to solve the task but also rotated the stimuli in the pictureplane (Cooper and Shepard, 1973). Although most studies examining mental rotation use three-dimensional figures, we could replicate reported brain activation patterns in adults (Cohen et al., 1996; Jordan et al., 2002; Richter et al., 2000, 1997). Therefore, our fMRI results in combination with results from other studies suggest that the brain areas associated with mental rotation are largely independent of the type of visual stimulus.

Observed activation in motor, premotor and supplementary motor areas (SMA) in children and adults brings up the possibility that also motor imagery could have been involved in this task. Characteristic features of the stimuli such as heads and bodies, as well as the fact that children could use manual rotation to check their answer during the practice trials may have induced a motor imagery strategy (Bonda et al., 1995; Funk et al., 2005; Wexler et al., 1998; Zacks et al., 1999, 2003). 


\section{Behavioral data}

Children and adults used mental rotation to solve the task. It took adults and children more time to solve the task when the stimuli had to be rotated $180^{\circ}$ compared to $90^{\circ}$ independent of the orientation in which the stimuli appeared. Therefore, increasing RTs with increasing rotation angle implies that participants used mental rotation to solve the task. We varied the stimulus orientation in such a way that the $180^{\circ}$ rotation trials where not necessarily associated with an upside-down stimulus presentation, which has been reported to sometimes elicit other types of strategies. The increase in time suggests that participants did in fact rotate the stimuli in the picture-plane (Cooper and Shepard, 1973). Our behavioral results are comparable to the findings of a recent study testing $90^{\circ}$ or $180^{\circ}$ mental rotation of colored drawings of different animals in children and adults (Jansen-Osmann and Heil, 2006). Consistent to our findings, RTs as a function of character orientation successfully served as a manipulation check that subjects indeed mentally rotated the stimuli. The fact that the increase in reaction time with increasing angle was more pronounced in children than in adults suggests that it is more difficult for children to rotate the stimuli mentally. Therefore, children needed more time to solve the problem, but they had no difficulty performing the task and they were using equivalent strategies to decide whether the two objects were identical or not. Several other studies confirm a continued overall decrease of reaction time with age, even in rather simple tasks, where young children and adults reach similar accuracy rates (Kwon et al., 2002; Rivera et al., 2005).

No practice effects were found from the first to the third block of condition. The reason for this might be the fact that every block contained a new type of stimuli. Consequently, new orientation and new strategy, which had to be acquired with every new block of stimuli inhibited effects of practice.

\section{Functional magnetic resonance imaging data}

An important point for the analysis of neuroimaging data in children is the choice of the statistical threshold. It is possible that assumptions about the ideal threshold for children may be overly conservative. Technical as well as developmental factors may influence the detection of the MR signal (Gaillard et al., 2001). For example, differential maturation rates in children could lead to greater interindividual variability. Moreover, many developmental studies provide a shift in patterns of cortical activation from diffuse to more focal activation of cortical regions with cognitive development [for review see (Casey et al., 2005)]. Therefore, we examined our children data at different thresholds to identify true activation extent not covered by any general differences in signal detection between children and adults. Using a lower statistical threshold in children revealed activation corresponding to the foci found in adults.

\section{Brain development}

Similar brain areas were activated in children and adults as reported earlier by Booth et al. (1999, 2000). Among those, the IPS is known to be a core region involved in mental rotation (Alivisatos and Petrides, 1997; Carpenter et al., 1999; Harris et al., 2000; Harris and Miniussi, 2003; Jordan et al., 2002; Kosslyn et al., 1998; Richter et al., 1997; Tagaris et al., 1996, 1998; Vingerhoets et al., 2001).

Adults vs. children

In the study presented here, the pattern in children was more lateralized to the right hemisphere in contrast to the adults' bilateral activation pattern. Several brain imaging studies in adults report bilateral parietal activation in mental rotation (Carpenter et al., 1999; Cohen et al., 1996; Jordan et al., 2002; Richter et al., 2000, 1997). Booth et al. (1999) also found a stronger right hemispheric activation in the right inferior parietal lobe (IPL) in children. In contrast to our findings they described a stronger left hemispheric activation in the superior parietal lobe (SPL) of children. We found that children activated the left IPS significantly less than adults. Observed differences between studies might be due to different kinds of mental rotation tasks and stimuli used (Vingerhoets et al., 2001). Moreover, activity in the left IPS correlated significantly with age and reaction time. This might reflect the important role of this region for effective and fast processing of visual manipulations. It is noteworthy, however, that adults beyond 30 years tended to show lower IPS activity compared to younger adults. To study this effect of age further we would need an increased sample size with more subjects older than 30 .

The developmental shift of brain activation in the IPS from a stronger right lateralized parietal pattern in children to the bilateral parietal pattern in adults could result from functional organization related to task performance changes during brain development. Processes of learning and expertise may account for such a change in laterality. For example, the left hemisphere has been shown to be engaged in well-trained cognitive tasks, while the right hemisphere is involved in the acquisition of skills needed to perform novel tasks (Goldberg and Costa, 1981). Moreover, a behavioral study 
revealed an advantage of the left hemisphere across blocks in a mental rotation task (Voyer, 1995). Different strategies are unlikely to be responsible for the difference in laterality because our results suggest that both children and adults used mental rotation to solve the task. Several imaging studies strengthen the idea of an increase in activity of the left hemisphere as an effect of training and practice. A PET study showed a linear increase of rCBF in the left hemisphere as an effect of practice in a spatial stimulus-response compatibility paradigm (Iacoboni et al., 1996). Moses et al. (2002) examined developmental changes in laterality for globally and locally directed analysis of forms. They concluded that during development, children show increasing left hemisphere advantage for local level processing resembling the pattern observed in adults. Moreover, the similar trend of hemispheric activation was found in the children cohort. Slow-performing children showed greater right hemispheric activation, thus contrasting the strong left hemisphere advantage of the adults. In the present study, however, only when merging children and adults, slow-performing participants show less activation in left IPS than fast-performing participants. Individual performance within the children and adult groups were not correlated with IPS activation in the left hemisphere. Therefore, individual levels of performance are unlikely to account for the brain activation pattern we found in this study.

The findings provide evidence for an increase in activation of the left hemisphere as a result of maturation. Furthermore, morphometric brain imaging studies show that maturation of gray matter and myelinization of frontal and parietal regions continue into adulthood (Gogtay et al., 2004; Sowell et al., 2003). Maturation and experience are tightly linked and hardly separable. Improved performance is highly correlated with increasing age in the majority of developmental studies (Allen and Ondracek, 1995; Kosslyn et al., 1990).

\section{Children vs. adults}

By a direct comparison of children vs. adults, differences in brain activation were found in medial occipital regions. Children showed no activation in the PCG and precuneus in comparison to adults, who showed a deactivation of these regions. These regions match closely to the so called 'default network', which was shown to decrease their activity during attention-demanding cognitive tasks (Raichle et al., 2001). We speculate that reduced or absent deactivation of this default network in children may explain less developed neuronal processing of goal directed behavior (Halder et al., 2006).

\section{Gender differences}

Gender differences in mental rotation favoring men have been noted in behavioral studies (Crucian and Berenbaum, 1998; Halpern, 2000; Linn and Petersen, 1985) and in functional imaging studies (Jordan et al., 2002; Tagaris et al., 1996; Thomsen et al., 2000; Unterrainer et al., 2000; Weiss et al., 2003).

The present study revealed no gender differences in the brain activation of children. Girls and boys seemed to use the same brain areas for mental rotation. These results also confirm other findings showing no differences between boys' and girls' EEG power values, whereas men exhibited more activation (lower EEG power values) than women in parietal and posterior temporal regions (Roberts and Bell, 2000, 2002).

In a previous study, we reported differences in brain activation patterns between women and men, although there were no differences concerning task performance (Kucian et al., 2005). In particular, women showed stronger activation in the right middle temporal gyrus, the right inferior frontal gyrus and the left primary motor cortex compared to men. These differences in cerebral processing might be attributed to different cognitive strategies of women and men while solving visuospatial problems (Jordan et al., 2002; Kucian et al., 2005).

Our findings suggest no difference in task performance between male (boys and men) and female participants (girls and women). Interestingly, several other studies also found no difference in performance between girls and boys before puberty (Karadi et al., 1999; Levine et al., 1999; Pezaris and Casey, 1991). It has to be noted, however, that Kerns and Berenbaum (1991) reported gender differences in children with boys outperforming girls. The authors argue that the failure of other studies to report gender differences may be due to the use of a paradigm design inappropriate for the assessment of mental rotation abilities in children. Kerns and Berenbaum (1991) used real 3D objects rather than pictorial $2 \mathrm{D}$ representations of objects. In our study, however, it is unlikely that the paradigm was unsuitable for children because pilot testing prior to scanning with 3rd grade school children ensured that the task can be solved correctly by young children. Moreover, the behavioral data we collected during scanning confirmed that children are accurate in this task. Thus, we think that the absence of any significant gender difference in children in our study cannot be attributed to the use of an inappropriate paradigm. However, it is conceivable that possible gender differences could unfold as mental rotation tasks become more difficult when more complex types of stimuli are used (e.g. 3D objects). In this 
context, it is noteworthy that the behavioral results we found in 6th graders could eventually result in a gender difference even though it was insignificant with the sample size we used in this study. Gender differences in mental rotation ability of children younger than thirteen years of age still remain controversial.

Yet another explanation for the differences in mental rotation task ability and fMRI could be the onset of puberty and the resulting hormonal changes. Not only do hormonal changes occur during puberty, but also the influence of prenatal androgen levels have been discussed with respect to gender differences in spatial abilities. For example a positive correlation between prenatal testosterone levels and performance scores of mental rotation in girls was reported (Grimshaw et al., 1995). In contrast, females with congenital adrenal hyperplasia (CAH, a genetic disorder that begins prenatally and causes overproduction of adrenal androgens) did not perform better than healthy females in mental rotation tasks (Hines et al., 2003). It would be interesting to include androgen levels in future developmental neuroimaging studies on spatial abilities in children. It is also possible that hormonal changes during puberty and the formation of gender specific strategies in mental rotation problem solving contribute to the observed differences in cerebral processing between women and men.

\section{Conclusion}

In conclusion, the present study suggests a continued specialization of brain areas into adulthood. Developmental changes of brain activation could be observed even though children and adults activated similar patterns during mental rotation. Maturation was leading to a bilateral activation in the IPS during mental rotation and faster performance.

\section{Acknowledgement}

We would like to thank our volunteers, especially the children, who participated in this study. This project was supported by the Neuroscience Center Zurich (ZNZ), the Hartmann-Müller Foundation for Medical Research Zurich and the National Center of Competence in Research (NCCR) on Neural Plasticity and Repair, Zurich.

\section{References}

Alivisatos B, Petrides M (1997) Functional activation of the human brain during mental rotation. Neuropsychologia 35(2): 111-118

Allen L, Ondracek P (1995) Age-sensitive cognitive abilities related to children's acquisition of spatial knowledge. Dev Psychol 31(6): 934-945

Bonda E, Petrides M, Frey S, Evans A (1995) Neural correlates of mental transformations of the body-in-space. Proc Natl Acad Sci USA 92(24): $11180-11184$
Booth J, Macwhinney B, Thulborn K, Sacco K, Voyvodic J, Feldman H (1999) Functional organization of activation patterns in children: whole brain fMRI imaging during three different cognitive tasks. Prog Neuro-Psychopharmacol 23(4): 669-682

Booth J, MacWhinney B, Thulborn K, Sacco K, Voyvodic J, Feldman H (2000) Developmental and lesion effects in brain activation during sentence comprehension and mental rotation. Dev Neuropsychol 18(2): 139-169

Carota A, Di Pietro M, Ptak R, Poglia D, Schnider A (2004) Defective spatial imagery with pure Gerstmann's syndrome. Eur Neurol 52(1): $1-6$

Carpenter PA, Just MA, Keller TA, Eddy W, Thulborn K (1999) Graded functional activation in the visuospatial system with the amount of task demand. J Cogn Neurosci 11(1): 9-24

Casey BJ (2002) Windows into the human brain. Science 296(5572): 1408-1409

Casey BJ, Tottenham N, Liston C, Durston S (2005) Imaging the developing brain: what have we learned about cognitive development? TRENDS Cogn Sci 9(3): 104-110

Cohen J (1988) Statistical power analysis for the behavioral sciences, 2nd edn. Lawrence Erlbaum, Hillsdale, NJ

Cohen MS, Kosslyn SM, Breiter HC, DiGirolamo GJ, Thompson WL, Anderson AK, Brookheimer SY, Rosen BR, Belliveau JW (1996) Changes in cortical activity during mental rotation. A mapping study using functional MRI. Brain 119 (Pt 1): 89-100

Cooper LA, Shepard RN (1973) Chronometric studies of the rotation of mental images. In: Chase WG (ed) Visual information processing. Academic Press, New York

Crucian GP, Berenbaum SA (1998) Sex differences in right hemisphere tasks. Brain Cogn 36(3): 377-389

Desrocher ME, Smith ML, Taylor MJ (1995) Stimulus and sex differences in performance of mental rotation: evidence from event-related potentials. Brain Cogn 28(1): 14-38

Dietrich T, Krings T, Neulen J, Willmes K, Erberich S, Thron A, Sturm W (2001) Effects of blood estrogen level on cortical activation patterns during cognitive activation as measured by functional MRI. NeuroImage 13(3): 425-432

Funk M, Brugger P, Wilkening F (2005) Motor processes in children's imagery: the case of mental rotation of hands. Dev Sci 8(5): 402-408

Gaillard WD, Grandin CB, Xu B (2001) Developmental aspects of pediatric fMRI: considerations for image acquisition, analysis, and interpretation. NeuroImage 13(2): 239-249

Gogtay N, Giedd JN, Lusk L, Hayashi KM, Greenstein D, Vaituzis AC, Nugent TF 3rd, Herman DH, Clasen LS, Toga AW, Rapoport JL, Thompson PM (2004) From the cover: dynamic mapping of human cortical development during childhood through early adulthood. Proc Natl Acad Sci USA 101(21): 8174-8179

Goldberg E, Costa LD (1981) Hemisphere differences in the acquisition and use of descriptive systems. Brain Lang 14: 144-173

Grimshaw GM, Sitarenios G, Finegan JA (1995) Mental rotation at 7 years: relations with prenatal testosterone levels and spatial play experiences. Brain Cogn 29(1): 85-100

Halder P, Brem S, Bucher K, Boujraf S, Summers P, Dietrich T, Kollias S, Martin E, Brandeis D (2006) Electrophysiological and hemodynamic evidence for late maturation of hand power grip and force control under visual feedback. Hum Brain Mapp (in press)

Halpern DF (2000) Sex differences in cognitive abilities, 3rd edn. Erlbaum, Mahwah

Harris IM, Egan GF, Sonkkila C, Tochon-Danguy HJ, Paxinos G, Watson JD (2000) Selective right parietal lobe activation during mental rotation: a parametric PET study. Brain 123 (Pt 1): 65-73

Harris IM, Miniussi C (2003) Parietal lobe contribution to mental rotation demonstrated with rTMS. J Cogn Neurosci 15(3): 315-323

Hines M, Fane BA, Pasterski VL, Mathews GA, Conway GS, Brook C (2003) Spatial abilities following prenatal androgen abnormality: 
targeting and mental rotations performance in individuals with congenital adrenal hyperplasia. Psychoneuroendocrinology 28(8): $1010-1026$

Iacoboni M, Woods RP, Mazziotta JC (1996) Brain-behavior relationships: evidence from practice effects in spatial stimulus-response compatibility. J Neurophysiol 76(1): 321-331

Jansen-Osmann P, Heil M (2006) Violation of pure insertion during mental rotation is independent of stimulus type, task, and subjects' age. Acta Psychol (Amst) 122(3): 280-287

Jordan K, Wustenberg T, Heinze HJ, Peters M, Jancke L (2002) Women and men exhibit different cortical activation patterns during mental rotation tasks. Neuropsychologia 40(13): 2397-2408

Kang HC, Burgund ED, Lugar HM, Petersen SE, Schlaggar BL (2003) Comparison of functional activation foci in children and adults using a common stereotactic space. NeuroImage 19(1): 16-28

Karadi K, Szabo I, Szepesi T, Kallai J, Kovacs B (1999) Sex differences on the hand mental rotation task for 9-yr.-old children and young adults. Percep Motor Skill 89(3 Pt 1): 969-972

Kerns KA, Berenbaum SA (1991) Sex differences in spatial ability in children. Behav Genet 21(4): 383-396

Klassencockpit (Ausgabe 2003/2004) MS_Deutsch_61, MS_Mathematik_ 61. Kantonaler Lehrmittelverlag St. Gallen, Rorschach

Klingberg T, Forssberg H, Westerberg H (2002) Increased brain activity in frontal and parietal cortex underlies the development of visuospatial working memory capacity during childhood. J Cogn Neurosci 14(1): $1-10$

Kosslyn SM, DiGirolamo GJ, Thompson WL, Alpert NM (1998) Mental rotation of objects versus hands: neural mechanisms revealed by positron emission tomography. Psychophysiology 35(2): 151-161

Kosslyn SM, Margolis JA, Barrett AM, Goldknopf EJ, Daly PF (1990) Age differences in imagery abilities. Child Dev 61(4): 995-1010

Kucian K, Loenneker T, Dietrich T, Martin E, von Aster MG (2005) Gender differences in brain activation patterns during mental rotation and number related cognitive tasks. Psychol Sci 47(1): 112-131

Kwon H, Reiss AL, Menon V (2002) Neural basis of protracted developmental changes in visuo-spatial working memory. Proc Natl Acad Sci USA 99(20): 13336-13341

Landerl K, Wimmer H, Moser E (1997) Salzburger Lese- und Rechtschreibtest. Verfahren zur Differentialdiagnose von Störungen des Lesens und Schreibens für die 1. bis 4. Schulstufe. Huber, Bern

Levine SC, Huttenlocher J, Taylor A, Langrock A (1999) Early sex differences in spatial skill. Dev Psychol 35(4): 940-949

Linn MC, Petersen AC (1985) Emergence and characterization of sex differences in spatial ability: a meta-analysis. Child Dev 56: 1479-1498

Marx H (1998) Knuspels Leseaufgaben. Gruppenlesetest für Kinder Ende des ersten bis vierten Schuljahres. Hogrefe, Göttingen

Moses P, Roe K, Buxton RB, Wong EC, Frank LR, Stiles J (2002) Functional MRI of global and local processing in children. NeuroImage 16(2): 415-424

Murphy K, Garavan H (2004) Artifactual fMRI group and condition differences driven by performance confounds. NeuroImage 21(1): 219-228

Peronnet F, Farah MJ (1989) Mental rotation: an event-related potential study with a validated mental rotation task. Brain Cogn 9(2): 279-288

Pezaris E, Casey MB (1991) Girls who use "masculine" problem-solving strategies on a spatial task: proposed genetic and environmental factors. Brain Cogn 17(1): 1-22

Raichle ME, MacLeod AM, Snyder AZ, Powers WJ, Gusnard DA, Shulman GL (2001) A default mode of brain function. Proc Natl Acad Sci USA 98(2): 676-682

Richter W, Somorjai R, Summers R, Jarmasz M, Menon RS, Gati JS, Georgopoulos AP, Tegeler C, Ugurbil K, Kim S (2000) Motor activity during mental rotation studied by time-resolved single-trial fMRI. J Cogn Neurosci 12(2): 310-320
Richter W, Ugurbil K, Georgopoulos A, Kim SG (1997) Time-resolved fMRI of mental rotation. Neuroreport 8(17): 3697-3702

Rivera SM, Reiss AL, Eckert MA, Menon V (2005) Developmental changes in mental arithmetic: evidence for increased functional specialization in the left inferior parietal cortex. Cereb Cortex 15(11): 1779-1790

Rivkin MJ (2000) Developmental neuroimaging of children using magnetic resonance techniques. Ment Retard Dev D R 6(1): 68-80

Roberts JE, Bell MA (2000) Sex differences on a mental rotation task: variations in electroencephalogram hemispheric activation between children and college students. Dev Neuropsychol 17(2): 199-223

Roberts JE, Bell MA (2002) The effects of age and sex on mental rotation performance, verbal performance, and brain electrical activity. Dev Psychobiol 40(4): 391-407

Schlaggar BL, Brown TT, Lugar HM, Visscher KM, Miezin FM, Petersen SE (2002) Functional neuroanatomical differences between adults and school-age children in the processing of single words. Science 296(5572): 1476-1479

Sowell ER, Peterson BS, Thompson PM, Welcome SE, Henkenius AL, Toga AW (2003) Mapping cortical change across the human life span. Nat Neurosci 6(3): 309-315

Stiles J, Moses P, Passarotti A, Dick FK, Buxton R (2003) Exploring developmental change in the neural bases of higher cognitive functions: the promise of functional magnetic resonance imaging. Dev Neuropsychol 24(2-3): 641-668

Tagaris GA, Kim SG, Strupp JP, Andersen P, Ugurbil K, Georgopoulos AP (1996) Quantitative relations between parietal activation and performance in mental rotation. Neuroreport 7(3): 773-776

Tagaris GA, Richter W, Kim SG, Pellizzer G, Andersen P, Ugurbil K, Georgopoulos AP (1998) Functional magnetic resonance imaging of mental rotation and memory scanning: a multidimensional scaling analysis of brain activation patterns. Brain Res Brain Res Rev 26(2-3): $106-112$

Thomsen T, Hugdahl K, Ersland L, Barndon R, Lundervold A, Smievoll AI, Roscher BE, Sundberg H (2000) Functional magnetic resonance imaging (fMRI) study of sex differences in a mental rotation task. Med Sci Monitor 6(6): 1186-1196

Unterrainer J, Wranek U, Staffen W, Gruber T, Ladurner G (2000) Lateralized cognitive visuospatial processing: is it primarily gender-related or due to quality of performance? Neuropsychobiology 41: 95-101

Vingerhoets G, Santens P, Van Laere K, Lahorte P, Dierckx RA, De Reuck J (2001) Regional brain activity during different paradigms of mental rotation in healthy volunteers: a positron emission tomography study. NeuroImage 13(2): 381-391

von Aster MG (2001) ZAREKI (Neuropsychological test battery for number processing and calculation in children). Swets \& Zeitlinger, Frankfurt am Main

Voyer D (1995) Effect of practice on laterality in a mental rotation task. Brain Cogn 29(3): 326-335

Weiss E, Siedentopf CM, Hofer A, Deisenhammer EA, Hoptman MJ, Kremser C, Golaszewski S, Felber S, Fleischhacker WW, Delazer M (2003) Sex differences in brain activation pattern during a visuospatial cognitive task: a functional magnetic resonance imaging study in healthy volunteers. Neurosci Lett 344: 169-172

Wexler M, Kosslyn SM, Berthoz A (1998) Motor processes in mental rotation. Cognition 68(1): 77-94

Wijers AA, Otten LJ, Feenstra S, Mulder G, Mulder LJ (1989) Brain potentials during selective attention, memory search, and mental rotation. Psychophysiology 26(4): 452-467

Zacks JM, Rypma B, Gabrieli JD, Tversky B, Glover GH (1999) Imagined transformations of bodies: an fMRI investigation. Neuropsychologia 37(9): 1029-1040

Zacks JM, Vettel JM, Michelon P (2003) Imagined viewer and object rotations dissociated with event-related FMRI. J Cogn Neurosci 15(7): 1002-1018 